\title{
Enfermería en el pasado y presente de la familia mexicana
}

\section{Nursing in the past and present of the mexican family}

Lic. Evertina Ramírez Díaz•

\section{Resumen}

Pocas estructuras de la vida social tienen tanta influencia sobre la personalidad como la familia, sin embargo, nada tan difícil como construirla y modelarla. En ella, el individuo se hace persona, desarrolla lo humano y se socializa para alcanzar los valores, las normas y las conductas que le permiten convivir de manera aceptable dentro de su grupo social. En su interior se forman los hábitos relacionados con la salud, se generan procesos que actúan como protectores de salud o desencadenantes de la enfermedad. La familia ha experimentado cambios muy significativos a lo largo de la historia, que han generado diversas formas de organización familiar. Es importante que las enfermeras reconozcan las repercusiones de la composición y estructura en la salud familiar, pues términos como familia nuclear, familia monoparental, de madre soltera, familia extendida, o familia homosexual, se refieren a personas viviendo en el mismo espacio familiar. Esta diversidad de familias tan heterogéneas, en cuanto a su naturaleza y a su desarrollo cotidiano, hace necesario que la Enfermera Familiar las considere al proporcionar atención a la familia, englobando los aspectos de su experiencia, como: su historia, su progreso y su estado actual, utilizando de forma prioritaria las estrategias de atención primaria.

Profesor de Carrera T.C Titular "A” ENEO-UNAM

GRUPO DE INVESTIGACIÓN EN CRONICIDAD Y BIENESTAR

Camino Viejo a Xochimilco y Viaducto Tlalpan San Lorenzo Huipulco CP 14370 México DF

correo electrónico: evertiram@yahoo.com.mx

Fecha de Recibido: 12 Marzo 2012

FeCha de EnViado: 31 Marzo 2012

Fecha de Aprobado: 22 MAYo 2012 


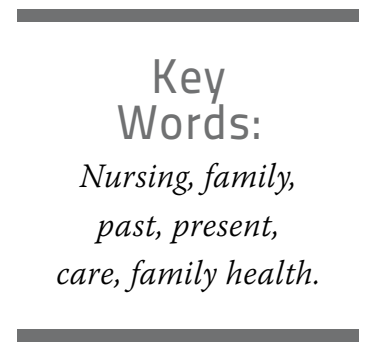

ABSTRAC

Few structures of social life have so much influence on personality as the family, however, not as difficult as building it and shaping her. Therein, the individual becomes person, develops human and socialize to achieve the values, norms and behaviors that allow you to live together in an acceptable way within your social group. Inside health-related habits are formed, generate processes that act as health protectors or triggers for the disease. The family has undergone significant changes throughout history, which have generated various forms of family organization. It is important that nurses recognize the impact of the composition and structure on family health, because terms like nuclear family, single-parent family, single parent, extended family, or family gay, refer to people living in the same family space. This diversity of families so heterogeneous, with regard to their nature and their everyday development, makes it necessary that the family nurse considered to provide care for the family, encompassing aspects of his experience, such as: its history, its progress and its current state as a priority using primary care strategies.

\section{INTRODUCCIÓN}

Pocas estructuras de la vida social tienen tanta influencia sobre la personalidad como la familia, sin embargo, nada tan difícil como construirla y modelarla; cómo hacer que fructifique positivamente lo que la familia puede dar a sus miembros y a la sociedad. La integración de la pareja conyugal es el principio de la familia, que se inicia cuando dos individuos distintos que pertenecen a diferentes grupos sociales, se separan de su núcleo original con la intención de formar una nueva familia. ${ }^{1}$ Alrededor de este grupo, considerado como la unidad básica de la sociedad, se mueven todos los individuos de una forma $u$ otra.

En la familia, el individuo se hace persona, desarrolla lo humano y se socializa para alcanzar los valores, las normas y las conductas que le permiten convivir de manera aceptable dentro de su grupo social. Asimismo, en el seno de esta, desarrolla la capa- cidad de relación con sus iguales, su identidad personal sobre la base de la identidad del grupo social al que pertenece, adquiere las habilidades necesarias para participar e integrarse en los distintos roles sociales; expresa así la maduración emocional y social obtenida a través del desarrollo de todas y cada una de las funciones que ejerce la familia. ${ }^{2}$ Los padres llevan impresos en su carácter y en sus actitudes, los valores, creencias y aspiraciones, así como las prohibiciones que prevalecen en el estrato social al que pertenecen, transmitiéndolos a sus hijos. ${ }^{3}$

El sistema familiar, también refleja en sí mismo, los grandes problemas de la sociedad en que vivimos, esta unidad de relaciones humanas depende de influencias tanto internas como externas y se relaciona prácticamente con todos los problemas y dimensiones de la vida humana.

\section{Cambios psicosociales EN LA FAMILIA}

La familia ha experimentado cambios muy significativos a lo largo de la historia, actualmente, en las distintas sociedades prevalecen muy variadas maneras de organización familiar. Con su evolución histórica, ha experimentado transformaciones que le han permitido adaptarse a la exigencia de cada sociedad y cada época, lo que hace prever que la familia persistirá, según Minuchin ${ }^{4}$, debido a que constituye la mejor unidad humana para sociedades rápidamente cambiantes. Cuanta mayor flexibilidad y adaptabilidad requiera la sociedad de sus miembros, más significativa será la familia como órgano del desarrollo psicosocial.

En el transcurso de una cuantas décadas, la sociedad ha manifestado profundas transformaciones, las cuales son más notables en el interior de las familias: cambios del 
papel de la mujer en el hogar, cambios en el ejercicio de la autoridad, en la crianza y educación de los hijos, agudización de los conflictos generacionales, quebranto del sentido de identidad y de las ligas de afecto que den cohesión al grupo familiar. $^{3}$

\section{Familia tradicional}

Hace no muchos años, cuando México salta de su pasado semifeudal basado en una economía agrícola y comercial, la familia bien integrada tenía una estructura jerárquica y estratificada, su dinámica estaba determinada por los roles en función de las diferencias sexuales de cada integrante. El proveedor, con la máxima autoridad; las mujeres subordinadas a los hombres y los jóvenes a los adultos; la interdependencia económica entre los miembros era estrecha.

Una de las transformaciones más importantes a fines del siglo $\mathrm{xx}$, ocurre al cobrar las mujeres consciencia de sus posibilidades de una vida más amplia y replantean su lugar tanto en la familia como en la sociedad, lo que ha generado una modificación de su posición frente al hombre, no como un ser dependiente ni con una sola misión dentro del hogar -como madre y ama de casa- sino como persona con igualdad de derechos, ${ }^{2}$ el efecto en la dinámica familiar no puede dejar de sentir y de requerir un cambio de la estructura familiar.

Desde los años cincuenta del siglo pasado, la inconformidad con esta situación se ha manifestado más abiertamente y tiende a generalizarse, de manera especial, entre las mujeres que han tenido una educación más liberal, que la que recibieron sus madres. Es innega- ble, que en la sociedad patriarcal tradicional, la mujer sufrió grandes discriminaciones, fue sometida por el hombre a servidumbre física, económica y psicológica, de tal manera, que cada vez es mayor la proporción de mujeres que buscan su liberación, a través de la educación y el trabajo fuera del hogar, para desarrollar una identidad propia y una vida más auténtica en el seno de una familia menos autoritaria y opresiva.

\section{Participación de la mujer en el campo laboral}

El origen de la participación económica de la mujer y su impacto en las relaciones familiares y de pareja se ubica en diferentes ámbitos y niveles de la realidad; situaciones económicas difíciles hace que se fomenten diferentes mecanismos de producción cotidiana, la política de población lleva a intensificar la reducción de la fecundidad y nuevas pautas reproductivas, todo ello, probablemente, ha creado condiciones más favorables para el trabajo extradoméstico femenino. Asimismo, se registran cambios en la dinámica familiar, en la relación de pareja y en la posición de ser mujer, el acceso a mayores niveles educativos y la progresiva seguridad jurídica de la mujer frente al hombre. ${ }^{5}$

Las evidencias indican que, por lo general, las mujeres desarrollan múltiples estrategias para combinar la maternidad y la actividad laboral: buscan trabajos que permiten flexibilidad de horarios, a poca distancia del hogar, o que pueden realizarse en casa, para no descuidar las funciones de ama de casa; utilizan el apoyo de familiares, amigos y vecinos; si es posible, contratan servicio doméstico remunera- do, y en algunos sectores, hacen uso de las estancias infantiles. Estas estrategias dependen del tipo de trabajo realizado, de las características de la familia y del apoyo que recibe por parte de la pareja. ${ }^{6}$

Según datos del Instituto $\mathrm{Na}$ cional de Estadística y Geografía, las madres mexicanas aportan el 42 por ciento del PIB nacional. Las mujeres con más de 15 años y por lo menos con un hijo, registran una tasa de participación del $42.2 \%$ en la economía, de las cuales, el 97.7\% también realiza actividades domésticas. Con base en la Encuesta Nacional de Ocupación y Empleo, en el segundo trimestre de 2011, el 96\% de las mujeres con hijos participaba en la producción de bienes y servicios (población ocupada). El $34.1 \%$ de las madres trabajaba en empresas y negocios y el $34.2 \%$ en el sector informal. La mayoría de mujeres que no tenía hijos laboraba principalmente en empresas y negocios. ${ }^{7}$

\section{Cambios en la estructura familiar}

Existe una gran diversidad de composiciones familiares, en donde se desarrolla la vida familiar, que va desde la tradicional familia nuclear y la familia extensa, hasta estructuras familiares en la que se cuenta con uno solo de los progenitores, o las familias con hijos adoptivos, familias en la que el padre y la madre están casados por segunda vez y tienen hijos de relaciones anteriores, en la que se hace presente la convivencia con padrastro o madrastra, la pareja que cohabita sin ningún contrato matrimonial; las compuestas únicamente por la pareja (conyugal), padres divorciados que no conviven con sus hijos. Asi- 
mismo, la diversidad sexual se viene incorporando en el concepto de familia, viéndose de forma más frecuente y aceptada en las sociedades modernas que conforman la familia homosexual, con o sin hijos adoptivos, y que comparten la expresión y el compromiso sexual. ${ }^{8}$ Estas formas de convivencias son algunos de los ejemplos que dan cuenta de las transformaciones que ha sufrido el modelo de familia.

Un cambio relevante en la estructura de la familia, es la posición del padre en cuanto autoridad. En generaciones pasadas, era común que el padre exigiera de sus hijos respeto y obediencia. Hoy la regulación de autoridad ha recaído en la mujer, la observación más frecuente es que el padre ha pasado del autoritarismo, a la falta de autoridad.

El centro de gravedad, ha girado hacia la madre, quien se ha convertido en la máxima autoridad del hogar, estableciéndose una relación no balanceada entre ésta y los hijos, ya que el padre, sólo cumple funciones externas; está poco en casa, no es reconocido por sus hijos como guía, por lo que ellos, difícilmente se identifican con él, lo que trae como consecuencia, la necesidad insatisfecha de autoridad, que se expresa en la conducta social de muchos jóvenes, a través de su ambivalencia hacia toda jerarquía institucional. $^{3}$

\section{Hogares monoparentales}

Bumpass, señala que el matrimonio como institución, refleja los cambios que han ocurrido en la sociedad. En la actualidad, se ha incrementado el número de hogares monoparentales, el que se constituye por uno de los padres y sus hijos, los datos estadísticos reportan un incremento en el número de hogares donde el jefe de familia es una mujer. En el año 2000, era de $4.6 \mathrm{mi}-$ llones, en 2005 de cada 100 hogares 23 estaban a cargo de mujeres. ${ }^{9}$ Este tipo de familia puede tener su origen en muy diversos factores, como la viudez femenina, el aumento en la proporción de madres solteras, los esquemas migratorios principalmente de hombres, el incremento de la separación y el divorcio, como producto de las crisis que se presentan en el marco de las interacciones conyugales, en la cual, los integrantes continuarán manifestándose de manera diferente, en la mayoría de los casos, el progenitor solo, se encuentra en una situación muy vulnerable, teniendo que hacer frente a responsabilidades; tanto en calidad de proveedor del sustento como cuidador de la familia.

Las tasas de divorcio han sido consideradas como indicadores de la estabilidad familiar y social, el INEGI informó que en México los divorcios presentan una tendencia a la alza de cerca de $4 \%$ anual, pues en 1980, por cada 100 matrimonios hubo 4 divorcios, en 1992 se alcanzó la cifra de 51 953, lo que indica una relación divorcios-matrimonios de 7.8; en el 2000, esta cifre se eleva a 7 divorcios, ${ }^{10}$ en 2006 se registraron 72396 divorcios, una relación de $12.3^{9}$ y para 2008 son casi 14 divorcios por cada 100 matrimonios. ${ }^{10}$

Con la disolución matrimonial, se permite la formación de nuevos matrimonios, los hijos casi siempre se ubican con la madre divorciada, pero eventualmente pueden seguir al padre. En cualquier caso, esto lleva a la formulación de una nueva familia o incluso el desplazamiento de los hijos con abuelos o tíos, situación que puede generar, diversas alteraciones de índole emocional, pues los hijos, tienen necesidades afectivas que deben ser satisfechas, como: mantener con un adulto una liga durable y confiable, recibir y dar amor, sentirse amparado ante los obstáculos y alentado en sus logros, etcétera, ya que en el proceso de responder a estas necesidades de los hijos, la familia modela su personalidad. ${ }^{3}$ Además pueden estar presentes, sentimientos de culpa, inseguridad, tristeza, comportamientos de rebeldía, agresividad, conductas antisociales, lo que los convierte en individuos muy vulnerables a factores como: adicciones, pandillerismo, embarazo en adolescentes, promiscuidad sexual, intento de suicidio, entre otros.

Esta diversidad de circunstancias origina familias muy heterogéneas en cuanto a su naturaleza y a su desarrollo cotidiano, que deberá tener en cuenta la enfermera al proporcionar atención a la familia.

\section{Participación de la enfermera en la atención familiar.}

Las enfermeras constituyen el recurso esencial para atender la salud de la familia, sin embargo es una tarea compartida. Cuidar desde un enfoque familiar implica incluirla en el proceso de prestación de cuidados, ya que es el entorno donde se establece por primera vez el comportamiento y las decisiones en materia de salud, de tal manera, que el contexto familiar brinda la posibilidad de tratar las necesidades de salud de la unidad familiar y de cada uno de sus miembros. ${ }^{11}$ Aunque la familia ha delegado algunas de sus funciones, continúa asumiendo la función principal de cuidado y protección de sus integrantes, ya que la percepción de una alteración de la 
salud, las decisiones y las acciones de cuidado comienzan y se desarrollan en el marco familiar. ${ }^{12}$

Por ello, las enfermeras reconocen a la familia como la unidad básica de las intervenciones de enfermería, y como señala Friedemann, se debe comprender que la estructura familiar puede representar un proceso dinámico, más que una variable estática, con diversas repercusiones en el estado de salud familiar.

Actualmente, la enfermera se enfrenta a una mayor pluralidad de formas familiares y de alternativas que cubren las diversas necesidades de los individuos, ya que la familia está cambiando en respuesta a las necesidades de sus miembros $\mathrm{y}$ a las presiones del entorno y de la sociedad. Es importante que las enfermeras conozcan y entiendan las repercusiones de la composición y estructura en la salud familiar, pues términos como familia nuclear, familia monoparental, de madre soltera o familia extendida, se refieren a personas viviendo en el mismo espacio familiar. ${ }^{13}$ La enfermera, más allá de la estructura familiar, orientará a la familia a mantener una estabilidad, a través de promover y hacer consciente a los integrantes de ésta, de la importancia del cumplimiento de normas, reglas y roles definidos, así como una comunicación abierta, clara y permanente para mantener el equilibrio de la salud familiar.

Cuando se aborda a la familia en procesos preventivos, promocionales y de rehabilitación, se están brindando cuidados de enfermería tendientes a lograr la salud familiar, la cual hace referencia "tanto al estado de salud de los integrantes de la familia, como a la efectividad o funcionalidad de la familia como unidad"14

Para Horwitz y cols. salud familiar, es el resultado del ajuste o equilibrio entre elementos internos y externos del grupo familiar, la capacidad de la familia para adaptarse y superar las crisis durante su ciclo. Así, la salud familiar puede considerarse, como el ajuste o equilibrio entre los elementos internos y externos del grupo familiar, incluyendo el estado de salud física y mental individual, así como el nivel de interacción entre los miembros de la familia. ${ }^{15}$

El estudio y atención de la familia, exige de la enfermera, conocimientos profundos en Enfermería Familiar, ha de reconocer la relación que hay entre la salud de la persona y la salud de la familia, de tal forma que al proporcionar cuidados a los distintos miembros, sanos o enfermos, la enfermera debe abarcar, todos los aspectos de la experiencia de la familia: su historia, su progreso, su estado actual y su futuro. Esto es posible, a través de la utilización en la práctica, de diversos modelos de atención, que le permita lograr el propósito central de las acciones de cuidado con este grupo, un abordaje holístico tanto de la familia como de cada uno de sus miembros y el logro de una relación que involucre a la enfermera-individuo-familia, sin dejar de considerar en las intervenciones, los aspectos psicosociales que prevalecen en la familia.

Las enfermeras, señala Friedemann, cuentan con una ventaja importante en relación a otros profesionales al proporcionar cuidados, pues ha participado en los eventos más significativos de la vida de la familia, como el nacimiento, el de- sarrollo humano en todas las etapas de la vida, las crisis, los estados de salud y enfermedad, la muerte, así como en las emociones básicas de éstas como, la ansiedad y la pena.

La enfermera en el ámbito de la salud familiar, puede diversificar sus acciones desde el contexto de la Atención Primaria en Salud, cuyo propósito principal se enmarca en la modificación y desarrollo de estilos de vida en las familias, así como aquellas que presentan problemas de salud más complejos en uno o más de sus integrantes, sin dejar de considerar, que tanto en uno como en otro contexto, la familia está inmersa en un ambiente sociocultural, posee una historia de vida que determina muchos de sus hábitos de comportamiento y de cuidado.

Las enfermeras que cuentan con formación, conocimientos, capacidades y comprensión de las distintas formas de familia, pueden favorecer a la familia sana fortaleciendo su estructura y sus funciones, así como la promoción de una dinámica familiar positiva que favorezca su salud.

Al prestar cuidados a las familias, se debe respetar el carácter único de cada una de ellas. Los estereotipos de algunas estructuras familiares, como las parejas del mismo sexo, pueden sentirse rechazadas y apartarse de toda búsqueda de apoyo, con lo que se incrementan sus riesgos de problemas de salud.

Es muy significativo, la forma en que la enfermera se acerca a la familia para ofrecer o brindar cuidado, pues se requiere de características y valores que le permitan ser aceptada en el contexto familiar, a través del reconocimiento de sus habilidades y capacidades en el 
abordaje de diferentes problemas por los que atraviese la familia.

Una de las alternativas con que cuenta la enfermera para realizar el abordaje de la familia, es a través de la aplicación del Modelo de Enfermería de Familia, ${ }^{16}$ el cual permite especificar e interpretar la información que se obtiene de ella, para aproximarse a la compleja realidad que incide en su salud. Este modelo está organizado en seis dimensiones configurativas que representan los aspectos constituyentes de la salud familiar: a) contexto socioeconómico y cultural; b) composición del hogar; c) procesos críticos de vida familiar; d) relaciones intrafamiliares; e) afrontamiento familiar y $f$ ) apoyo social.

\section{- El contexto socioeconómico} y cultural de la vida familiar, permite obtener datos sobre los aspectos socio-laborales de la familia, la situación económica y las condiciones materiales de vida del hogar, así como el aspecto cultural en el cual se desarrolla la vida cotidiana de la familia, como es la religiosidad y los valores ético - morales.

- Composición del hogar, incluye las características de la estructura familiar que comparte un hogar como son el número de convivientes, las relaciones de parentesco entre ellos y con relación a un jefe del hogar, la edad y el sexo.

- Procesos críticos de vida familiar, hace referencia a la vivencia en la familia de acontecimientos de diferente naturaleza que afectan la estabilidad y el funcionamiento en un determinado período. Los acontecimientos normativos, paranormativos y de salud, suponen valoraciones en las relaciones familiares, en los sentimientos, se generan exigencias y cambios en su funcionamiento interno. ${ }^{16}$

- Relaciones intrafamiliares, este apartado permite valorar la calidad (armoniosa, estable, disfuncional) de las relaciones (interpersonales e intrafamiliares) entre sus miembros, ya que con frecuencia pueden constituirse en un problema cuando no se establecen los suficientes vínculos afectivos. Cada familia establece sus prototipos de relacionarse entre sí, lo que impactará en el desarrollo físico, intelectual, psicológico y espiritual de cada uno de sus integrantes.

- Afrontamiento familiar, implica la capacidad que tiene el grupo familiar de movilizarse, de tomar decisiones ante los problemas y el modo de comportarse frente a los procesos críticos, puede ser constructivo cuando se generan actitudes y comportamientos favorables a la salud como la flexibilización de normas establecidas, ajuste de roles, solicitan apoyo comunitario o profesional; y destructivo, cuando la familia evade el problema y asume conductas de negación y rechazo, evitando el tratamiento y/o apoyo de cualquier índole.

- Apoyo social, constituye el conjunto de recursos de diversa naturaleza con los que pueden contar las familias en la vida cotidiana $y$ en otros momentos en que no está en posibilidades de hacer frente a determinadas situaciones al rebasar sus recursos y potencialidades. Los tipos de apoyo pueden ser instrumental, material o psicológico. $^{16}$

Se debe tener en cuenta que las nociones de la realidad que estas dimensiones representan se interrelacionan en forma sistémica integrando lo biológico, lo social y lo psicológico en el contexto familiar, de tal forma que el modelo proporciona a la enfermera una concepción de salud del grupo familiar y una perspectiva integradora de los procesos que la involucran, fortaleciendo la visión entre la relación familia-salud-enfermera.

Por la relevancia innegable que tiene la enfermera en el trabajo con familia, la OMS ha señalado, que la enfermera de familia "ha de contar con conocimientos profundos sobre la complejidad, tanto de las personas, familias y grupos, como de las interacciones que se producen entre ellos. Así mismo, ha de conocer la comunidad donde ofrece servicios, los recursos disponibles y las diferentes maneras de interactuar entre sus miembros. La enfermera de familia necesita también estar preparada para intervenir en órganos de salud pública, ya que ella puede identificar elementos en el barrio o en la comunidad que influyen de manera positiva o negativa en la salud de la familia y las personas que la componen. Deberá ser una activa participante".

\section{Conclusiones}


La familia transmite de generación en generación, su historia, su experiencia, sus valores, sus costumbres, elementos relacionados estrechamente con la obtención de salud. En su interior se forman los hábitos relacionados con la salud, se generan procesos que actúan como protectores de salud o desencadenantes de la enfermedad, asimismo se desarrollan recursos de apoyo muy significativos. El funcionamiento familiar constituye un factor predisponente en la producción y curso de diversas enfermedades y problemas de salud en sus integrantes.

La familia como sistema dinámico, se transforma de acuerdo a su contexto social y político en el que se desenvuelve, aunque su organización básica se mantiene como familia nuclear, los cambios en la estructura familiar han sido evidentes. Puede creerse que la diversidad de las formas de familia constituye una pérdida de los valores familiares tradicionales, pero quizá es un indicativo, de que la familia es en realidad muy resistente en su adaptación a las nuevas condiciones sociales.

En la actualidad se reconoce que las condiciones psico-sociales, económicas y políticas impactan de manera notable en el estado de salud de las personas, de la familia y de la comunidad, por ello la importancia de la participación de la enfermera con enfoque familiar que le permita revalorar a la salud familiar como forma de proporcionar el cuidado con sentido crítico, humano y holístico.

La enfermera, debe reconocer la relación que existe entre el funcionamiento de la familia y el estado de salud de los integrantes que la componen en todas sus áreas, para que pueda intervenir de manera eficaz y con responsabilidad, utilizando de forma prioritaria las estrategias de la atención primaria en salud.

Al trabajar con la salud familiar, la enfermera, debe considerar al conjunto de los integrantes donde interviene, el funcionamiento familiar, la estructura, la forma en que enfrentan las etapas del ciclo vital, el modo de vida de la familia (factores socioeconómicos, socioculturales, sociopsicológicos, psicosociales), las potencialidades de la familia (recursos protectores) y las del medio (sistema de salud, redes de apoyo) para poder tener una visión amplia, de la situación específica de la salud familiar que se manifestará en el estado de salud, de riesgo o de daño de la misma.

\section{REFERENCIAS BIBLIOGRÁFICAS}

1 Eguiluz L. (Compiladora) Dinámica de la familia. Un enfoque psicológico sistémico. México: Pax; 2003 p. 9.

2 Novel MG. et al. Enfermería Psicosocial y Salud Mental. Serie Manuales de Enfermería, Barcelona: Masson; 2000 p.67-72.

3 De la Fuente R. Psicología Médica, México: Fondo de Cultura Económica, 2004 p.154-157.

4 Minuchin S. Familias y terapia familiar. Barcelona: Gedisa, 1983.

5 Vidaurri J. Mujeres que trabajan y relación de pareja. Un análisis desde la perspectiva de género. En: Dulanto E. La familia. Un espacio de encuentro y crecimiento para todos. México: Editores de textos mexicanos, 2004, p. 17-20.

6 García B. y Oliveira O. Trabajo femenino y vida familiar en México, México, El Colegio de México, 1994 p. 19

7 INEGI. Encuesta Nacional de Ocu- pación y Empleo 2011. México 2012. Disponible en: http://bit.ly/ SXiwX3

8 González E. Psicología del ciclo vital. Madrid, España: Editorial CCS. 2da. ed, 2002 p. 225-287.

9 INEGI. Censo General de Población y Vivienda 2010. Tipo y clase de Hogar. México 2011. Disponible en: http://bit.ly/TSLatb

10 INEGI. Estadísticas de Matrimonios y Divorcios 2008. México. Disponible en: http://bit.ly/Ug49uo

11 OPS-OMS. La familia y la salud. CD44/10 Consejo directivo. $55 \mathrm{Se}$ sión del comité regional. Septiembre 2003 Disponible en: http://bit. ly/VB7vHV

12 Pardo TM. Familia y Cuidado. En: Dimensiones del Cuidado. Grupo de cuidado. Facultad de Enfermería. Universidad Nacional de Colombia. Santafé de Bogotá. D.C. 1998 p 125-131.

13 Friedemann ML. The Framework of systemic organization. A conceptual approach to families and nursing. USA: Sage Publications; 1995.

14 Amaya de Peña P. Familia de ancianos y escolares del sector de la Estrella, Ciudad Bolívar (fase diagnóstica), Bogotá. Facultad de Enfermería. Universidad Nacional de Colombia, 1992 p. 12.

15 Horwitz N, Florenzano R, Ringeling I. Familia y Salud Familiar. Un enfoque para la Atención Primaria. Bol Of Sanit Panam 1985; 98(2): 144-55. Disponible en: http://bit. ly/12jGheJ

16 Louro BI. Modelo de salud del grupo familiar. Rev Cubana Salud Pública, 2005; 31 (4): 332-7 Disponible en: http://bit.ly/RnGJrs 